\title{
ALTERNE: Intelligent Virtual Environments for Virtual Reality Art
}

\author{
Marc Cavazza $^{1}$, Jean-Luc Lugrin ${ }^{1}$, Simon Hartley ${ }^{1}$, Paolo Libardi ${ }^{1}$, Matthew J. \\ Barnes $^{1}$, Mikael Le Bras ${ }^{1}$, Marc Le Renard ${ }^{2}$, Louis Bec ${ }^{3}$ and Alok Nandi ${ }^{4}$ \\ ${ }^{1}$ School of Computing, University of Teesside, Middlesbrough TS1 3BA, United Kingdom \\ m.o.cavazza@tees.ac.uk \\ ${ }^{2}$ CLARTE, 6 rue Léonard de Vinci, BP 0102, 53001 Laval CEDEX, France \\ Marc.LeRenard@esiea-ouest.fr \\ ${ }^{3}$ CYPRES, Friche de la Belle de Mai, 41 rue Jobin, 13003, Marseille, France \\ info@cypres-artech.org \\ ${ }^{4}$ Commediastra, 182, av. W. Churchill, 1180 Brussels, Belgium \\ nandi@architempo.net
}

\begin{abstract}
Virtual Reality Artworks provide sophisticated user experiences but their development is a complex process. We describe the use of Intelligent Virtual Environments for VR Art. In this approach, virtual world behaviour is governed by a symbolic system. This supports news forms of experimentation with physical laws or with causality that we have termed Alternative Reality. In addition, basing world behaviour on a symbolic representation can facilitate collaboration between artists and scientists. We introduce the techniques behind Alternative Reality and illustrate these with examples from the prototype. We conclude by an outline of artistic work in progress using these techniques.
\end{abstract}

\section{Introduction}

Virtual Reality Art [1] has proven to be one of the most challenging applications of Virtual Reality (VR). At the same time, artistic installations have provided some of the most convincing VR experiences. However, the development of VR Art installations is a complex process, even more so considering the necessary collaboration between artists and scientists. If the artistic creation of virtual worlds is to proceed by "ways of worldmaking", one natural question is to determine the conceptual level describing these new worlds. This theoretical question echoes the more pragmatic one, which seeks to improve the practical collaboration between artists and scientists in the actual development of VR Art installations. The global framework for this research is the ALTERNE project, an EU-funded project aiming at developing new technologies for VR Art, following an Art+Science approach [2]. The project is based on the concept of Alternative Reality, which revisits the early ideas of VR according to which its purpose was to provide psychedelic experiences [3] rather than being an accurate simulation of physical reality.

In the next sections, after a brief presentation of the system's architecture, we describe the technology baseline for Alternative Reality, and how their symbolic 
nature provides a new framework for the collaborative design by artists and scientists. As the artistic work is currently in progress, we conclude by giving a brief outline of how artistic briefs will be making use of this technology.

\section{System Overview and Architecture}

In order to maximise user experience, Virtual Reality Art is often presented using immersive displays such as CAVEs ${ }^{\mathrm{TM}}$. Our target systems are large-scale virtual reality installations, such as the SAS Cube ${ }^{\mathrm{TM}}$, which is a 4-wall, PC-based, CAVETM_ like, immersive visualisation system. The use of a CAVETM-like system should facilitate interaction with virtual world objects, which is an essential aspect of the alternative reality experience. We achieve this interaction through a game engine, Unreal Tournament $2003^{\mathrm{TM}}$ (UT) that is utilized both as a visualisation engine and as a development environment. Game engines are now increasingly used for visualisation in scientific research due to their rendering performance and their ability to communicate with external software modules [4], which in the present case is essential to the development of a simulation layer that will override basic physics mechanisms. Another interesting aspect is the growing use of game engines for $3 \mathrm{D}$ Digital Arts.

In addition, the engine we are using, UT, has previously been ported to CAVETM systems [5] and we have adapted it to the SAS Cube ${ }^{\mathrm{TM}}$, using the original approach described in the CAVE-UT implementation. Figure 1 illustrates the display of one of our test environments in the SAS-Cube ${ }^{\mathrm{TM}}$.

This environment supports several interaction mechanisms in terms of object manipulation (which can be grabbed, moved, so as to initiate physical processes), as well as "triggers" that can start certain processes associated with the environment's devices (heating, cooling, flowing, etc.). Game engines such as UT 2003 ${ }^{\mathrm{TM}}$ include sophisticated behaviour models, which can be broken down into i) event systems that manage user interaction and discretise physical processes and ii) native physics engine supporting realistic simulation. This feature provides an essential path to the implementation of new behavioural layers, which is the technical principle behind the implementation of alternative reality.

\section{AI Techniques for World Behaviour}

While it is accepted that AI techniques can support virtual agents behaviours, there are fewer applications in which they support the behaviour of the world as a whole, substituting themselves to physical simulation. However, this situation changes dramatically when the objective is to depart from physical realism, i.e. to create alternative worlds obeying different laws of physics. There is a significant history of VR Art creating alternative worlds, such as Osmose ${ }^{\mathrm{TM}}$ from Char Davies. Challenging the laws of physics has even been the plot for one of the most popular pieces of animation, The Quarxs ${ }^{\mathrm{TM}}$, by Maurice Benayoun. Let us consider the 
objective of creating alternative worlds in which different laws of physics would apply, or in which causality would be modified. The main conceptual difficulty consists in creating these alternative world behaviour's from a principled basis. Devising mathematical formulations for alternative laws of physics would be highly unpractical, if at all feasible. Rather, physical behaviour could be described at a higher level of abstraction, in terms of the qualitative relations between physical phenomena that constitute physical laws.

There is an obvious advantage in using symbolic descriptions of behaviour. These can be embedded in a knowledge level whose concepts serve as a basis for discussion between artists and scientists. Although this level is not free from formalisation, it is still possible to identify explicit concepts within the AI representations (in the present case, derived from Qualitative physics or Planning) that it uses.

This section describes the fundamental mechanisms developed to describe alternative reality. Their instantiation to actual artistic briefs will be presented in the next section.

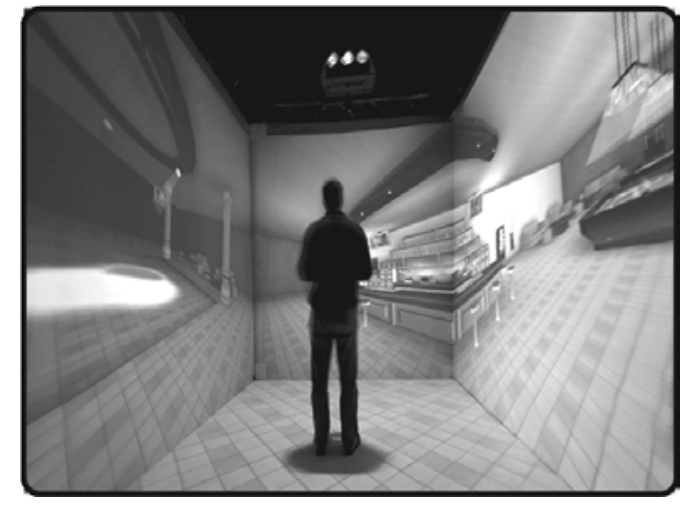

Figure 1: Our Test Environments (the Causality Café) in the SAS Cube ${ }^{\mathrm{TM}}$.

\subsection{Redefining World Behaviour through an Event-based System}

The mechanism underlying the definition of new worlds' behaviours relies on the event system which is associated to the UT engine. This provides a natural discretisation of physical events which supports the overriding of the native physics engine by AI-based behavioural engines. Our architecture is based on an Event Interception System (EIS) that defines specific classes of objects, which can enter a specific event interception mode [6]. In this mode events involving the object (e.g. the object breaking into pieces when being hit) can have their activation delayed. There exists explicit representation for the elementary events (that we refer to as Basic Events), which are low-level events derived from the collision detection mechanisms of the graphic engine, e.g. Bump (Actor OtherActor), Touch(Actor OtherActor), UnTouch (Actor OtherActor), 
ActorEnteredVolume (Actor Volume), etc. From these basic events, more abstract events are instantiated, by encapsulating the associated representations as well as relevant properties of the objects they involve.

The EIS underlies both the Qualitative Physics Engine and the Causal Engine. In the former case, objects that can take part in qualitative processes intercept physical events involving them so as to allow their behaviour to be under the control of qualitative simulation; basic events are parsed into QP-events that can activate relevant processes. In the latter case, intercepted events can be modified to create causality-inducing event co-occurrences; basic events are parsed into Context Events (CE). For instance, the basic events hit(?obj1, ?obj2) and explode(?obj2) can be encapsulated into a CE_break_object(?obj1, ?obj2). As the explosion effect has been intercepted, the corresponding CE is termed "frozen". This status allows a whole range of transformation prior to its re-activation, which is the basis for the creation of artificial causal impressions.

Finally, basic events are associated animations visualizing their effects. They also constitute the basis for the integration between the discretise event system and the visual effects in the virtual environment.

\subsection{From Qualitative Physics to Alternative Physics}

Qualitative Physics [7] has been developed for the symbolic simulation of physical processes. We have extended its use to the definition of alternative physical laws, which support the definition of alternative world behaviour on a principled basis. The principle behind qualitative physics is to make discrete the variation of physical properties and to model all physical transformations through processes that encapsulate the relation between physical variables, through the notion of influence equations (see details of process described below). The qualitative physics engine is implemented in an external $\mathrm{C}++$ program that communicates with the UT $3 \mathrm{D}$ environment [8].

We can illustrate alternative physics through the definition of a fluid flow process which describes the filling of a glass, which is allowed to contain more fluid than its volume would normally allow:

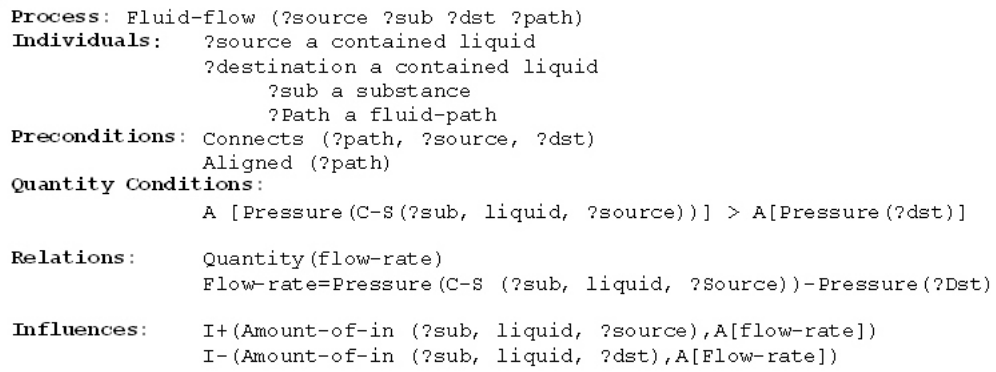




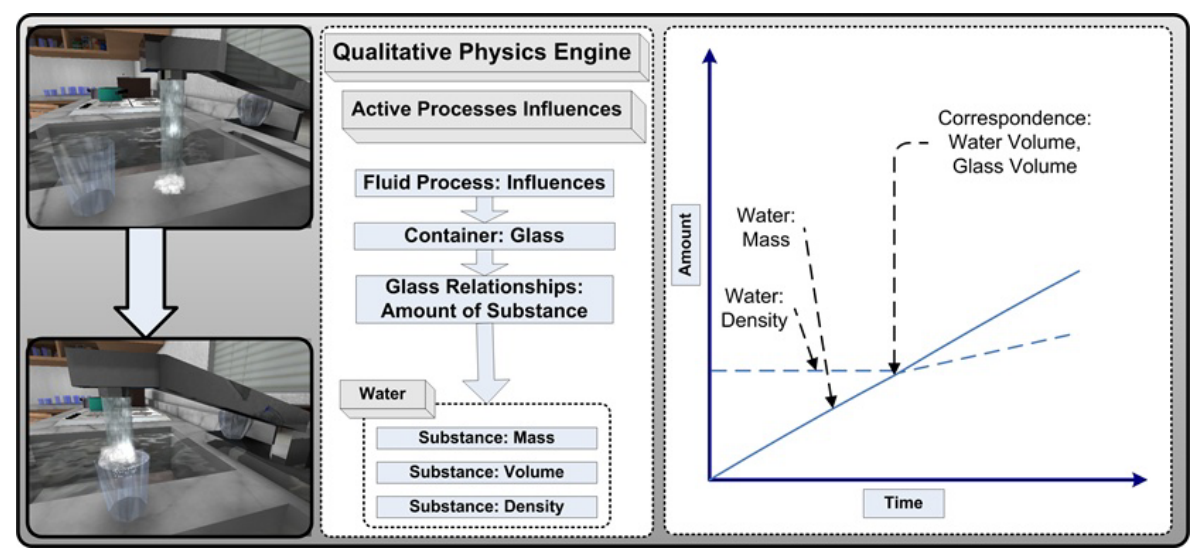

Figure 2: Example of a Qualitative Process in Action: The Alternative "Filling" Process.

In our example, the filling process increases the amount of water in the glass due to the existence of a water flow from the tap. However the qualitative equations that govern the progression of mass and volume can be dissociated so that, when a certain landmark value is reached, the glass' mass increases independently of its volume. Eventually a limit point for the glass/container mass will be reached and this will determine a new behaviour for the filled glass, such as the glass exploding under the amount of water contained.

Recently, we have extended qualitative modelling to the simulation of physiological systems. In this context, it supports the simulation of life forms, including imaginary ones, which are the subject of the Arapuca artistic project (see below).

\subsection{Modifying Causality}

Causality is an important aspect of how we construct reality. This makes it an ideal target for alternative experiences. There exists several psychological theories aiming at explaining the attribution of causal relations between co-occurring events, starting with Michotte [9]. From an empirical perspective, our objective was to create a system in which the consequences of a given action (generally initiated by the user) could be modified to follow not the "natural" laws of causality but different principles.

The causal engine operates by modifying the Context Events produced by regularly sampling events in the virtual world (which are triggered by processes and/or user intervention). We previously referred to the CEs as "frozen", as their effects are temporarily inhibited. CEs are represented using a STRIPS-like model [10], which makes explicit the pre-condition for the event and its post-conditions in the form of procedural changes to the world. In that representation, there is very much a cause-effect relation between Basic Events detected in the pre-condition and effects triggered in the post conditions. 
The basic mechanism for creating "artificial" co-occurrences consists in modifying a CE's post-conditions, after the basic events corresponding to its pre-conditions have been observed. These modifications are performed while the CE is "frozen" through the application of specific Macro-operators (henceforth MOps), which are applied to the STRIPS-like representation of CEs.

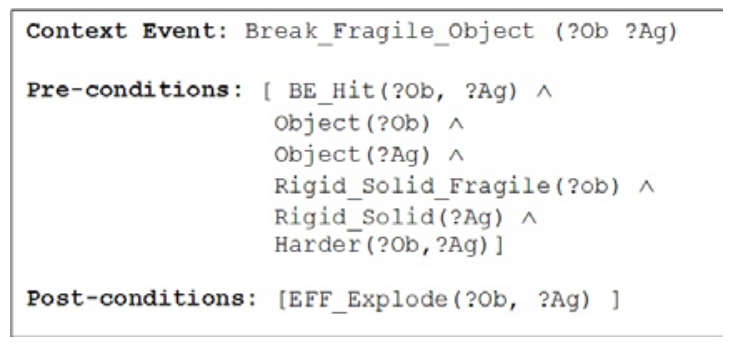

We have developed a test environment, the Causality Café, which includes a numerous objects that can be part of various physical events (containers can be open or filled, objects can be thrown or fall, can be pushed to roll or slide, can be broken, devices can be activated, doors or elements of furniture can be open, etc.). Physical processes can be embedded naturally into relevant artefacts such as taps, beer pumps and a pool table. For the example we describe here we've been using 58 object instances from 11 object categories (e.g. container, divider) and 9 types of effects (i.e. Break, Bounce, Tilt). The starting event is the user throwing a bottle to the glass door of a refrigerator containing similar bottles. The normal (expected) effect would be for the thrown bottle to break on impact and/or the fridge door itself to break.

The first step when the causal engine receives the "Break Fragile Object" CE from the EIS is to determine the best candidate transformations. Among the transformations considered to generate alternative effects are the substitution of the CE's objects (which would result in different objects being broken than the one which was hit) and the propagation of the CE's effects to other objects. Every MOp uses semantic compatibility measures to produce a heuristic value from objects comparisons, effects comparisons and to check the applicability of alternative effects on the CE's objects. One such effect is the propagation of the "break" effect to beer bottles inside the fridge, of the same kind as the original (i.e. throwing a bottle at the fridge would break bottles inside it without breaking the fridge's door or penetrating the fridge, see Figure 3). The search process can produce a whole range of alternatives (Figure 3), such as another exploding that the one being hit by the bottle.

More importantly, the strategies for creating such co-occurrences can be controlled using specific types of MOp (for instance, based on analogy). The use of such concepts to direct the strategy is also a basis for discussion between artists and scientists. 


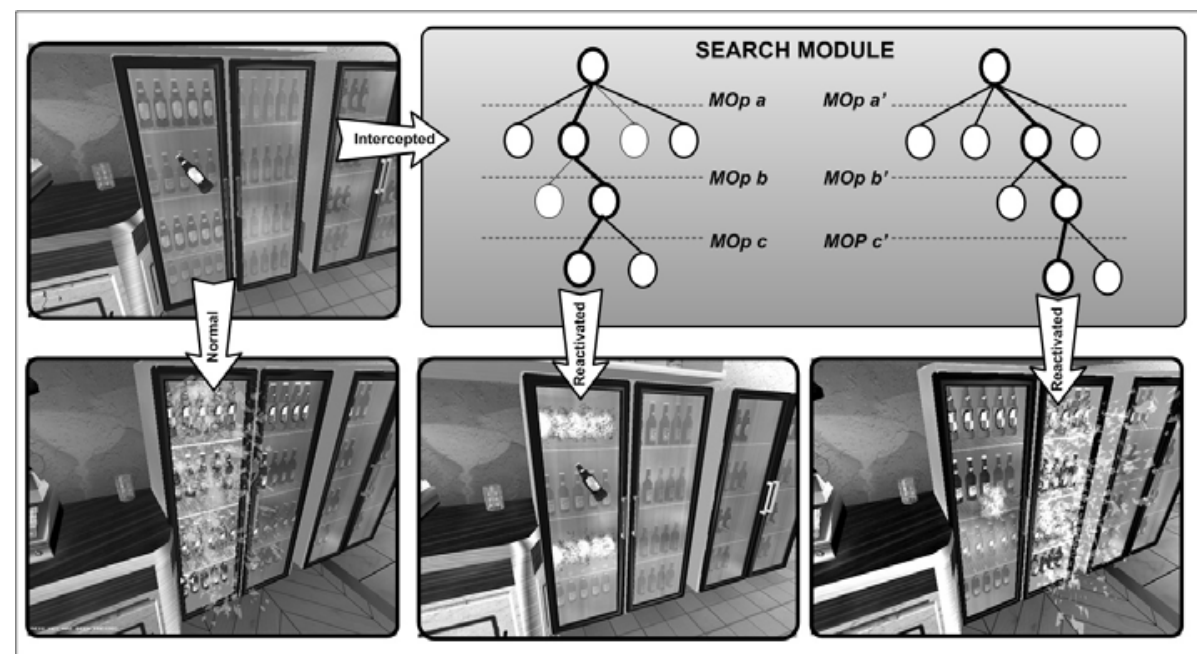

Figure 3. The Causal Engine in Action (see text).

\section{Example Briefs}

In this section we give a brief outline of artistic work in progress which takes advantage of the concept of alternative reality.

\subsection{Arapuca: Artificial Life Forms in Artificial Ecosystems}

This briefs revisits previous work in Digital Arts and Artificial Life [11] with the new tools provided by the ALTERNE approach. It supports the real-time simulation of alternative life forms (the Upokrinomena; in our case the specimen is a Diaphaplanomena) by grounding their behaviour on a model of their internal physiological processes, which is based on Qualitative Physiology. Physical processes in the environment in which the creature evolves (e.g. heat transfer, currents, turbulences, etc.) are also modelled using qualitative physics, hence resulting in the simulation of a complete imaginary ecosystem. Dynamic interactions between the Diaphaplanomena in its environment (such as the creature entering a cold current or being hit by turbulence) generate events that are passed to the simulation engine and alter the current simulation. For instance (Figure 4), if the creature is hit by a turbulence, its course will be modified (physical simulation), which will prompt the Diaphaplanomena to correct its trajectory (physiological simulation) with effect in the environment (physical simulation). A first prototype for this brief has been implemented. 


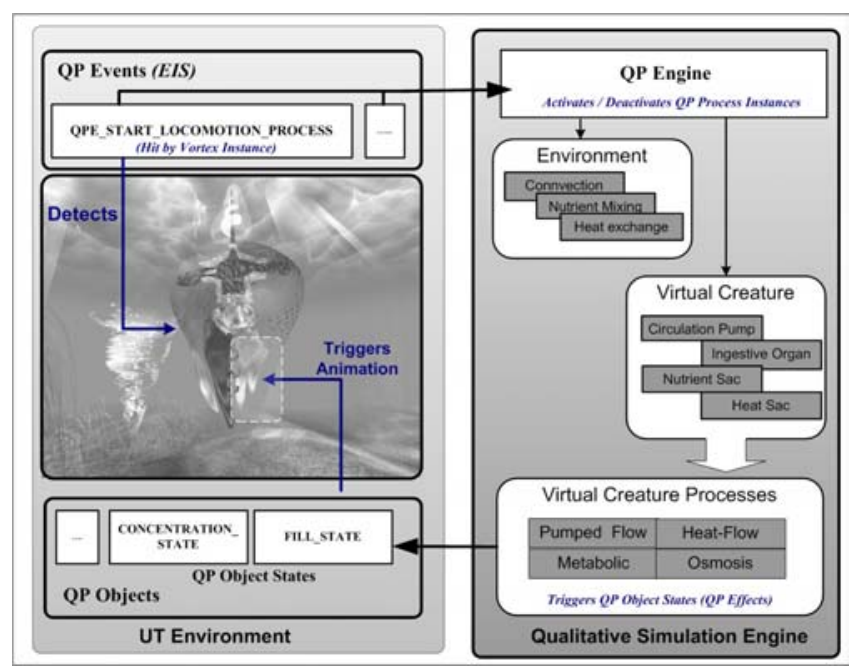

Figure 4: The Diaphaplanomena in its Ecosystem.

\subsection{Ego.Geo Graphies: Blurring the line between Objects and Actors}

This artistic brief is also situated in an imaginary world with alternative laws of physics. One of its key elements is that it features the behaviour of physical objects as part of a virtual narrative, thus blurring the line between objects and actors in a narrative. This brief is an exploration of the notion of context through the variable behaviour of virtual actors and the environment as an actor, responding to the user involvement in the environment. As Figure 5 illustrates, this brief features a certain number of object-actors being emitted by certain areas of the landscape. These acquire the status of autonomous agents, whose main interaction mode with other agents (or with elements of the virtual world) is physical collision. On the other hand, these episodes of interaction are determined by artistic authoring: in particular, the consequences of these interactions should be made to vary according to the narrative context, which is itself influenced by user interaction. Having introduced these elements, we can now relate this brief to our alternative causality system, especially considering the role of collisions and their consequences, which have previously served to illustrate the basic mechanisms of our causal engine. In this brief the effects of collisions will be governed by several high-level factors such as the "empathy" demonstrated by the user towards certain object-actors. In reference to the above described mechanisms, the effects can consist in actors bouncing from obstacles, exploding, or being absorbed by them. In other cases, both colliding entities can disappear creating other objects such as vortices instead. Each collision will thus be associated a set of Context Events, whose post-conditions will be transformed by MOp determined by the current context. In other words, artistic concepts such as empathy, measured for instance by the extent of interaction with certain object categories, will govern causality, through the MOp selection process. The knowledge engineering process consists in identifying possible effects and, on the other hand, using numerical values derived from elements of narrative context as heuristics in the causal engine.

As such, the brief is highly relevant to the notion of Intelligent Virtual Environment since the behaviour of the environment as a whole acquires narrative status. This brief is largely under development at the present time. Figure 5 shows an overview of the brief's environment and some of its objects-characters. 


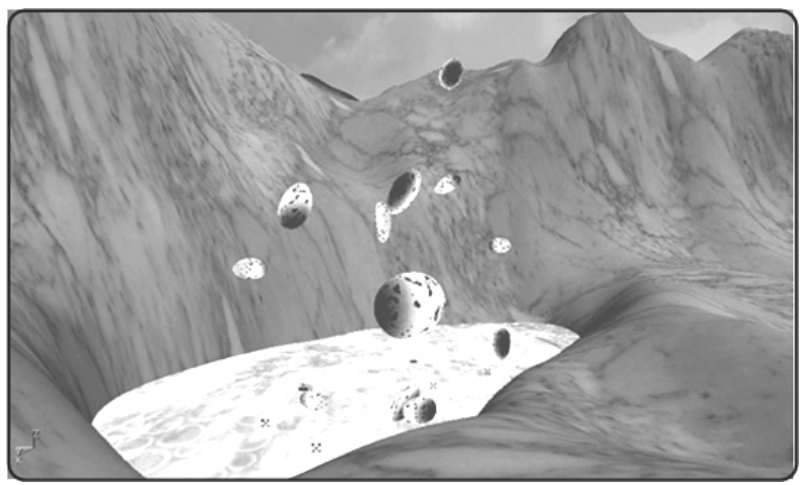

Figure 5: The World of "Ego.Geo Graphies".

\section{Conclusions and Perspectives}

VR Art, in its pursuit of imaginary worlds, raises interesting challenges for Virtual Reality Systems. In particular, the possibility of authoring world behaviour at a conceptual level is a major benefit that can be derived from Intelligent Virtual Environments. Our alternative reality approach introduces two AI-based behavioural techniques, one derived from Qualitative Physics and one inspired from planning techniques. This approach does not solve per se the difficult problem of developing software systems from artistic ideas. Rather, in line with its recourse to AI techniques, it takes steps to transform the software development process into a knowledge engineering approach. In that sense, while the direct manipulation of its formalisms by the artists is not a practical proposal, this approach facilitates discussion at a conceptual level, which is closer to the artistic ideas (Figure 6).

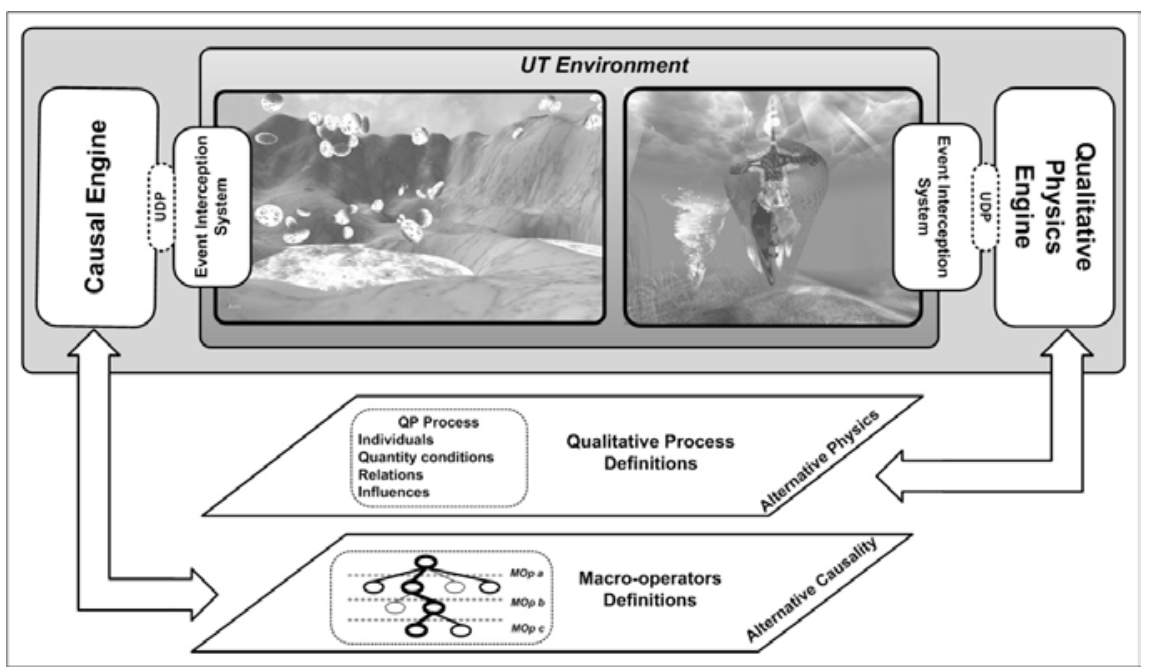

Figure 6. The Representational Layer Underlying Alternative Reality Worlds. 
At the same time, the conversion of such ideas into actual world behaviours is also facilitated and this enables fast prototyping and its correlate, quick feedback. Examples of this are for instance: i) the description of alternative laws of physics in plain English, which can then be converted into modification of qualitative processes and ii) the description of causal associations (co-occurrences between categories of events), which can be translated into corresponding search strategies in the causal engine on the basis of high-level concepts such as analogy.

\section{Acknowledgements}

The ALTERNE project (IST-38575) is funded in part by the European Commission, under the IST initiative (Cross-Programme Action 15). Sean Crooks is thanked for developing the visuals of Figure 5. Jeffrey Jacobson developed the original CAVEUT2003 system and is thanked for his assistance in adapting it to the SAS-Cube ${ }^{\mathrm{TM}}$.

\section{References}

1. Moser, M.A. (Ed.), Immersed in Technology: Art and Virtual Environments, Cambridge (Massachussets), MIT Press., 1996.

2. C. Sommerer and L. Mignonneau (Eds.),Art@ Science, New York: Springer Verlag, 1998.

3. Leary, T. Chaos and Cyberculture, Ronin Press, 1994.

4. Lewis, M and Jacobson, Games Engines in Scientific Research. Communications of ACM, Vol. 45, No. I, pp. 27-31, 2002.

5. Jacobson, J. and Hwang, Z. Unreal Tournament for Immersive Interactive Theater. Communications of the ACM, Vol. 45, 1, pp. 39-42, 2002.

6. Cavazza, M., Hartley, S., Lugrin, J.-L. and Le Bras, M., 2002. Alternative Reality: A New Platform for Digital Arts, ACM Symposium on Virtual Reality Software and Technology (VRST2003), pp. 100-108, Osaka, Japan, October 2003

7. Forbus, K.D., Qualitative Process Theory, Artificial Intelligence, 24, 1-3, pp. 85-168, 1984.

8. Cavazza, M., Hartley, S., Lugrin J.-L. and Le Bras, M., Qualitative Physics in Virtual Environments, ACM Intelligent User Interfaces, pp. 54-61, 2004.

9. Michotte, A., The perception of causality. New York:Basic Books. Translated from the French by T. R. and E.Miles, 1963.

10. Fikes, R. E. and Nilsson, N. J., STRIPS: a new approach to the, application of theorem proving to problem solving. Artificial Intelligence, 2 (3-4), pp. 189-208, 1971.

11. Bec, L., Elements d'Epistemologie Fabulatoire, in: C. Langton, C. Taylor, J.D. Farmer, \& S. Rasmussen (Eds.), Artificial Life II, SFI Studies in the Sciences of Complexity, Proc. Vol. X. Redwood City, CA: Addison-Wesley, 1991. 